\title{
FUNGOS ASSOCIADOS ÀS SEMENTES DE FUMO (Nicotiana tabacum L.)
}

\author{
SEGATO, Silvelena Vanzolini ${ }^{1}$ \\ GABALDI, Felipe Cravo ${ }^{2}$
}

RESUMO: O objetivo do trabalho foi verificar os fungos associados às sementes de fumo. Para tanto se utilizaram de sementes recém colhidas de frutos fechados ou abertos e sementes de frutos armazenados por um ano em ambiente frio. A sanidade foi avaliada pelo teste do papel de filtro "blotter test", utilizando 200 sementes com e 200 sementes sem desinfestação superficial para cada tratamento. Inicialmente as sementes foram divididas em duas porções e uma delas foi tratada com hipoclorito de sódio $2 \%$ durante um minuto (desinfestadas). As sementes sem desinfestação foram lavadas durante um minuto com água destilada esterilizada. Os resultados obtidos permitem concluir, que as sementes que foram desinfestadas com hipoclorito de sódio apresentaram redução no número de fungos encontrados nas sementes. Conclui-se que os fungos encontrados pertenceram aos gêneros: Fusarium, Curvularia, Colletotrichum, Cladosporium, Alternaria; Epicoccum e Nigrospora. Os fungos mais constantes nos tratamentos foram dos gêneros: Fusarium, Cladosporium e Alternaria. A menor incidência de fungos foi em sementes recém colhidas e de frutos abertos de fumo. A desinfestação superficial realizada com as sementes de fumo por um minuto com hipoclorito de sódio a $2 \%$ reduziu o no número de fungos avaliados.

Palavras-chave: Sanidade. Maturação. Sementes Recém Colhidas. Sementes Armazenadas. Desinfestação superficial com hipoclorito de sódio.

SUMMARY: The objective was to determine the fungi associated with seeds of tobacco. For that we used freshly harvested seeds of enclosed and open fruits, and seeds stored for one year in a cold environment. Sanity was evaluated by the filter paper blotter test, using 200 seeds and 200 seeds without surface disinfection for each treatment. Initially, the seeds were divided into two portions and one was treated with sodium hypochlorite $2 \%$ for one minute (disinfected). Seeds without disinfection were washed for one minute with sterile distilled water. The results showed that the seeds that were disinfected with sodium hypochlorite showed a reduction in the number of fungi found in the seeds. We conclude that the fungi found belonged to the genera: Fusarium, Curvularia, Colletotrichum, Cladosporium, Alternaria, Epicoccum and Nigrospora. Fungi were more constant in the treatments of the genera: Fusarium, Cladosporium and Alternaria. The lower incidence of fungi was in fresh seeds and fruits open fumo. A surface disinfection performed with seeds of fungi evaluated.

Keywords: Sanity, maturation, fresh seeds, seed storage, surface disinfection with sodium

\section{INTRODUÇÃO}

A espécie Nicotiana tabacum L. (fumo ou tabaco) pertence à família Solanaceae e é uma planta autógama, herbácea e anual que produz muitas sementes por fruto. O centro de origem provável é a América do Sul (AKEHURST, 1981; SEFFRIN, 1995)

Atualmente, o fumo é cultivado em todas as partes do mundo e é responsável por uma atividade econômica que envolve milhões de dólares (MEDEIROS, 2008).

O Brasil é referência mundial na qualidade do fumo, é o segundo maior produtor depois da China e o maior exportador mundial, principalmente devido à produção integrada e a produção de muitos tipos de fumo (ANUÁRIO..., 2009).

O tabaco pode ser usado de diversas maneiras, de acordo com sua forma de apresentação: inalado (cigarro, cachimbo, charuto e cigarro de palha), aspirado (rapé) e mascado (fumo de rolo). O setor

\footnotetext{
${ }^{1}$ Professor Doutor da Faculdade de Agronomia "Dr. Francisco Maeda"- FAFRAM/FE, Ituverava, SP e do Centro Universitário Moura Lacerda - CUML, Ribeirão Preto, SP. E-mail: sv.segato@ bol.com.br

${ }^{2}$ Graduando- Agronomia FAFRAM/FE, Ituverava, SP.
} 
fumageiro exerce grande importância na atividade econômica e social do Brasil. Na área econômica, o fumo é responsável pela arrecadação de grandes somas em impostos. No campo social, a atividade fumageira é grande geradora de empregos diretos e indiretos. No meio rural, o fumo ocupa a mão-de-obra de mais de 900 mil agricultores. As usinas de beneficiamento e as fábricas de cigarros empregam mais de 40 mil pessoas. No total, considerando a soma dos empregos diretos e indiretos gerados pelo fumo desde o seu plantio até a comercialização do cigarro, há o envolvimento de aproximadamente 2,4 milhões de pessoas que de alguma forma estão vinculadas ao setor. Para as mais de 191.000 famílias de agricultores, o fumo constitui-se na principal fonte de renda (AFUBRA, 2007).

A produção de sementes tem se convertido em uma atividade cada vez mais especializada, em face da necessidade do aperfeiçoamento e do desenvolvimento de novas e melhores técnicas, que permitam utilizar ao máximo as condições ecológicas favoráveis e o potencial de rendimento das espécies, dentro de padrões de qualidade física, fisiológica, sanitária e genética (MEDEIROS, 2008).

No Brasil, as sementes são produzidas pelas indústrias fumageiras e parte delas é vendida para os produtores e parte é exportada. Os produtores, por sua vez, são cooperados e produzem as folhas que são vendidas para a mesma empresa. Para a comercialização é obrigatório que as sementes tenham no mínimo $80 \%$ de germinação e $99 \%$ de pureza física (APASSUL, 2012) e, para o controle da qualidade das sementes são avaliadas a germinação, a pureza física e a sanidade.

De acordo com Hutchens (s/d), sob condições favoráveis de crescimento da planta-mãe, a semente de fumo - Nicotiana tabacum (L.) atinge a maturidade fisiológica ao redor de 21 a 24 dias após a fertilização do zigoto. Os índices de germinação e vigor de sementes colhidas são reduzidos, sendo que o autor enfatiza que as sementes de fumo fisiologicamente maduras necessitam gradativamente perder a umidade de forma natural, secarem a campo, antes de serem colhidas e estocadas.

Segundo Neergard (1979), diversos fungos quando presentes nas sementes podem provocar aborto, deformações, podridões, descolorações e necroses, com reflexos na diminuição da viabilidade e no vigor das mesmas.

De acordo com Miller (1995), os fungos que invadem sementes e grãos em geral são frequientemente divididos em dois grupos: fungos do campo, que infectam o produto ainda no campo e fungos de armazenamento, que invadem, no caso, o milho pouco antes e durante o armazenamento. A distinção entre fungos de campo e de armazenamento não é baseada na classificação taxonômica, mas de acordo com as condições ambientais e/ou ecológicas que favorecem o crescimento dos mesmos. Também não é absoluta, pois é baseada nos seus hábitos de crescimento e onde os danos ocorrem. Os fungos de campo requerem um grau de umidade em equilíbrio com uma umidade relativa de $90-100 \%$ para crescerem. Os principais gêneros são Cephalosporium, Fusarium, Gibberella, Nigrospora, Helminthosporium, Alternaria e Cladosporium que invadem grãos e sementes durante o amadurecimento e o dano é causado antes da colheita. Estes fungos não se desenvolvem normalmente durante o armazenamento, exceto em milho armazenado com alto grau de umidade (MILLER, 1995).

Os principais fungos envolvidos na perda de produtos armazenados são as várias espécies de Aspergillus e algumas espécies de Penicillium, os quais têm sua atividade regulada pelas condições ambientais ocorrentes durante o período de armazenamento e pelas condições do lote de sementes, especialmente de seu estado físico, grau de umidade e inóculo inicial (LUCCA-FILHO, 1985).

Contudo, para tal espécie é escassa a literatura sobre os fungos que a semente pode transportar e/ou transmitir. Assim, o objetivo principal do trabalho foi o de verificar quais os fungos associados às sementes de fumo. Procurou-se observar ainda se os fungos seriam diferentes em sementes recém colhidas de frutos fechados ou abertos e em sementes de frutos armazenados por um ano e ambiente frio e ainda 
saber se a desinfestação superficial com hipoclorito de sódio $2 \%$ durante um minuto poderia influir nos resultados do teste de sanidade (teste do papel de filtro).

\section{MATERIAL E MÉTODOS}

Foram utilizadas sementes coletadas em plantação de fumo no campus da FAFRAM no ano de 2008 e armazenadas em geladeira (dentro de saco plástico para manutenção da umidade e em baixa temperatura, aproximadamente $10^{\circ} \mathrm{C}$ ) e sementes recém colhidas no ano de 2009 de frutos abertos e fechados. Todos os três tratamentos foram secos a sombra por 3 a 4 dias.

A avaliação da qualidade sanitária das sementes foi realizada, utilizando-se o blotter test (DHINGRA; SINCLAIR, 1995). Para cada lote foram utilizadas 200 sementes com e 200 sem desinfestação superficial. Para cada amostra foram feitas quatro repetições de 50 sementes. Inicialmente as sementes de cada um dos três tratamentos foram divididas em duas porções e uma delas foi tratada com hipoclorito de sódio $2 \%$ durante um minuto (desinfestadas). As sementes sem desinfestação foram lavadas durante um minuto com água destilada. Por serem pequenas as mesmas foram manuseadas sobre uma peneira forrada com um tecido dito organza, evitando assim perdas. Foram utilizadas caixas de acrílico tipo gerbox previamente desinfestadas com álcool 70\%. Cada gerbox foi forrada com duas folhas de papel mata-borrão pré-umedecidas com água destilada.

Após sete dias de incubação a temperatura de $\pm 20^{\circ} \mathrm{C}+\mathrm{luz}$, as análises das sementes foram feitas individualmente ao microscópio estereoscópico verificando a incidência de fungos, e quando necessário para certificação dos resultados, foi feito o exame da morfologia dos fungos ao microscópio de luz para sua identificação, comparando com informações disponíveis na literatura (BARNETT; HUNTER, 1987).

Foi utilizado o delineamento experimental inteiramente casualizado. As repetições e o número de sementes analisadas estão na metodologia de cada teste realizado.

\section{RESULTADOS E DISCUSSÃO}

Na Figura 1 encontram-se os dados referentes à incidência de fungos em sementes de fumo armazenadas por um ano a baixa temperatura.

Figura 1 - Incidência de fungos (\%) em sementes de fumo armazenadas em condições de baixa temperatura. Ituverava, SP, FAFRAM, 2010.

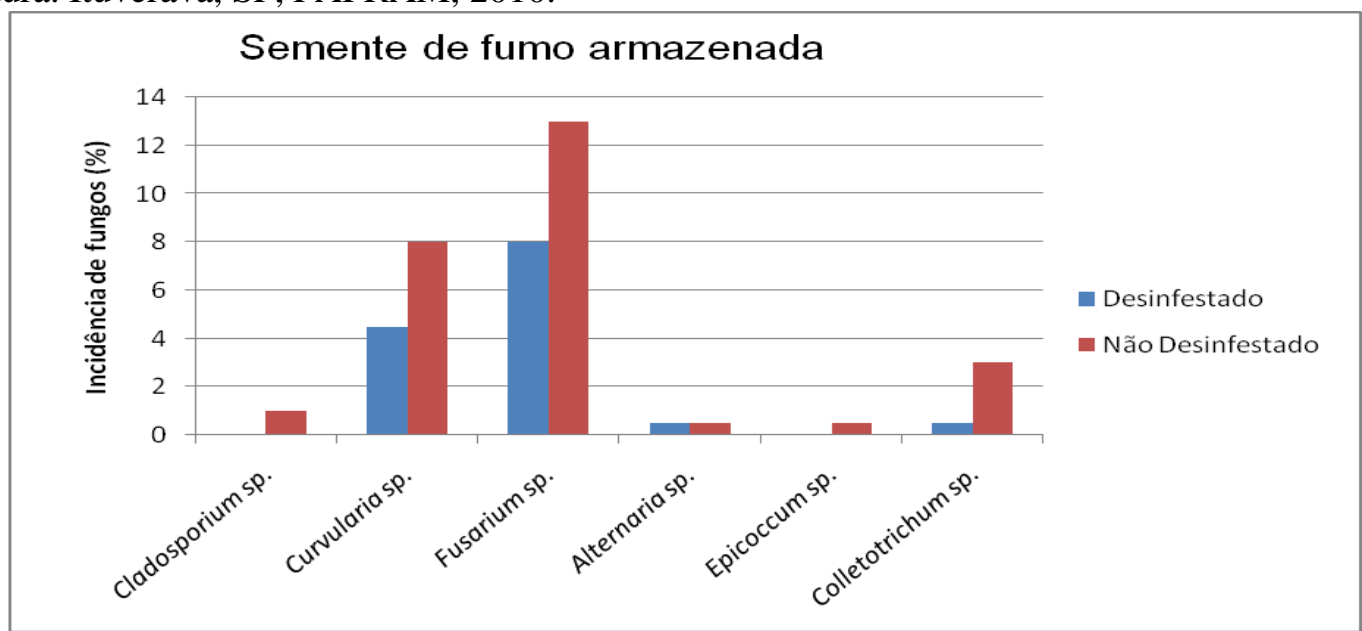

Fonte: Elaborado pelos Autores

Com base nos dados apresentados na Figura 1, pode-se observar que nas sementes de fumo armazenadas os fungos encontrados em ordem decrescente pertenceram ao gêneros: Fusarium, 
Curvularia, Colletotrichum, Cladosporium, Alternaria e Epicoccum.

Ainda verificou-se que as sementes de fumo armazenadas que foram desinfestadas superficialmente apresentaram, de forma geral, menor incidência de fungo que as não desinfestadas.

Na Figura 2 encontram-se os dados referentes à incidência de fungos em sementes de fumo recém colhidas de frutos ainda fechados.

Figura 2 - Incidência de fungos (\%) em sementes de fumo recém colhidas de frutos fechados. Ituverava, SP, FAFRAM, 2010.

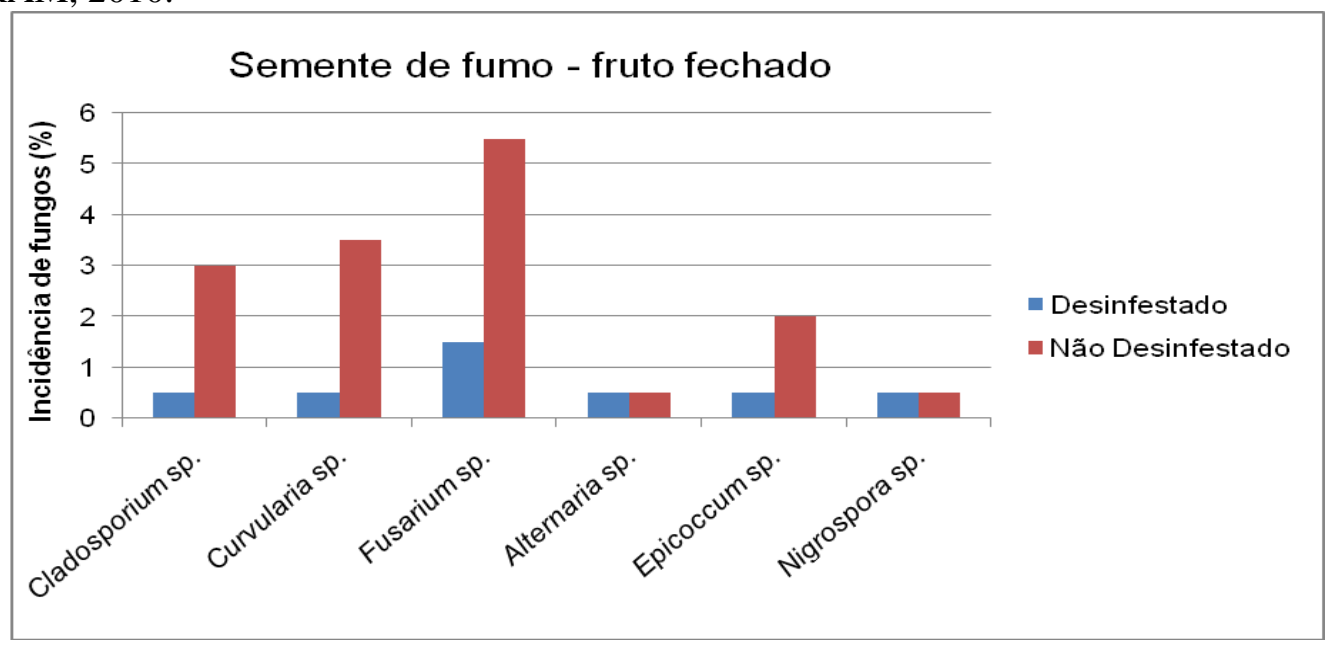

Fonte: Elaborado pelos Autores

Nos dados referentes à Figura 2, pode-se observar que nas sementes de fumo recém colhidas de frutos ainda fechados os fungos encontrados em ordem decrescente pertenceram aos gêneros: Fusarium, Curvularia, Cladosporium, Epicoccum, Alternaria e Nigrospora.

Em relação ainda aos resultados mostrados na Figura 2, nas sementes de fumo recém colhidas de fruto fechado quando foi feito o tratamento com hipoclorito de sódio a $2 \%$, observou-se que, de forma geral, houve menor incidência de fungos que as não desinfestadas, contudo para Alternaria sp. e Nigrospora sp. não houve diferença quanto ao tratamento.

Na Figura 3 encontram-se os dados referentes à incidência de fungos em sementes de fumo recém colhidas de frutos em início de abertura.

Figura 3 - Incidência de fungos (\%) em sementes de fumo recém colhidas de frutos abertos. Ituverava, SP, FAFRAM, 2010.

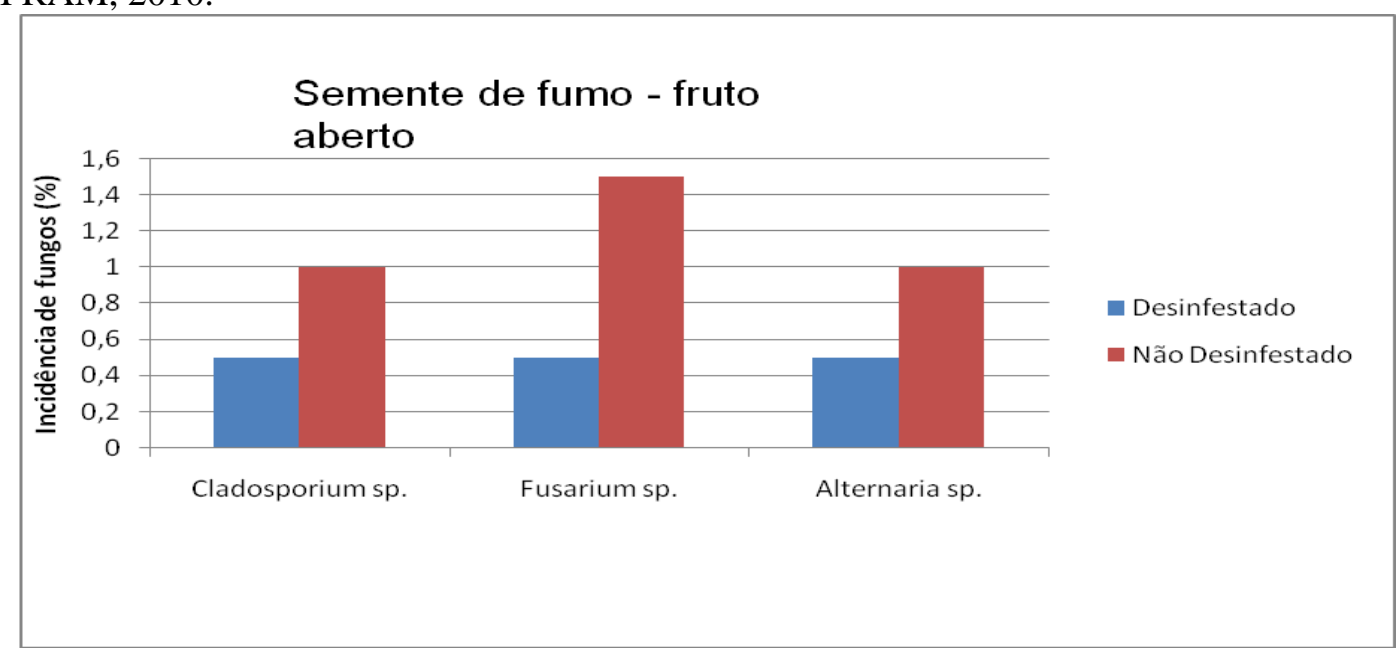

Fonte: Elaborado pelo Autor 
Em relação aos resultados mostrados na Figura 3, nas sementes de fumo recém colhidas de frutos abertos, os fungos encontrados em ordem decrescente pertenceram aos gêneros: Fusarium, Cladosporium e Alternaria.

Nos dados referentes à Figura 3, pode-se observar redução no número de Alternaria sp., Fusarium sp. e Cladosporium sp. com o tratamento realizado com hipoclorito de sódio a $2 \%$.

De modo geral comparando os três gráficos (Figuras 1, 2 e 3) verificou-se que os fungos encontrados pertenceram aos gêneros: Fusarium, Curvularia, Colletotrichum, Cladosporium, Alternaria e Epicoccum e Nigrospora e tais fungos podem apresentar potencial patogênico. Os fungos mais constantes foram dos gêneros: Fusarium, Cladosporium e Alternaria. De acordo com Miller (1995), os fungos que invadem sementes e grãos em geral são freqüentemente divididos em dois grupos: fungos de campo, que infectam o produto ainda no campo e fungos de armazenamento, que invadem a semente pouco antes e durante o armazenamento. Os fungos de campo requerem um grau de umidade em equilíbrio com uma umidade relativa de $90-100 \%$ para crescerem e dentre os principais gêneros o autor destacou as três espécies mais encontradas nesse trabalho: Fusarium, Alternaria e Cladosporium que invadem sementes durante o amadurecimento e o dano é causado antes da colheita. Segundo o mesmo autor estes fungos não se desenvolvem normalmente durante o armazenamento, exceto em sementes armazenadas com alto grau de umidade (MILLER, 1995).

Verificou-se também nesse trabalho que a menor incidência de fungos foi em sementes recém colhidas e de frutos abertos, seguido por frutos fechados e a maior incidência foi em frutos armazenados. Talvez a maior umidade das sementes de frutos ainda fechados e daquelas armazenadas em geladeira foi a causa da maior incidência de fungos, embora trata-se de uma suposição, visto que não foi realizado o teste para medir o grau de umidade das mesmas. Segundo Hutchens (s/d), sob condições favoráveis de crescimento da planta-mãe, a semente de fumo - Nicotiana tabacum (L.) atinge a maturidade fisiológica ao redor de 21 a 24 dias após a fertilização do zigoto. O autor enfatiza que as sementes de fumo fisiologicamente maduras necessitam gradativamente perder a umidade de forma natural, secarem a campo, antes de serem colhidas e estocadas. Portanto, as sementes de frutos abertos provavelmente secaram mais e, assim, apresentaram melhor qualidade sanitária.

Os fungos mais encontrados nesse trabalho do gênero Fusarium e até mesmo saprófitas como Alternaria e Cladosporium pode provocar descoloração das sementes. Segundo Lucca-Filho (1985) a descoloração de sementes é um fator de depreciação muito grande. No material destinado à semeadura este tipo de anormalidade caracteriza, geralmente, a presença de parasitas; nos produtos destinados ao consumo, geralmente são indicativos de uma baixa qualidade. Existe um número muito grande de fungos causadores de descoloração de sementes em diferentes culturas.

Ainda é possível inferir que a desinfestação superficial com hipoclorito de sódio resultou em menor infestação com os principais fungos verificados o que demonstra que boa parte da quantidade total de fungos estavam localizados no tegumento da semente e, portanto, foi oportuna a desinfestação visando avaliar realmente os fungos transportados internamente nas sementes.

\section{CONCLUSÃO}

De acordo com os resultados observados nesse trabalho, concluiu-se que em sementes de fumo:

- os fungos encontrados pertenceram aos gêneros: Fusarium, Curvularia, Colletotrichum, Cladosporium, Alternaria, Epicoccum e Nigrospora.

- os fungos mais constantes nos tratamentos foram dos gêneros: Fusarium, Cladosporium e Alternaria. 
- a menor incidência de fungos foi em sementes recém colhidas e de frutos abertos de fumo.

- a desinfestação superficial realizada com as sementes de fumo por um minuto com hipoclorito de sódio a $2 \%$ reduziu o número de fungos avaliados.

\section{REFERÊNCIAS}

AFUBRA - Associação dos Fumicultores do Brasil. Relatório de Atividades Afubra 2007. Santa Cruz do Sul: AFUBRA, 2007. 10p.

AKEHURST, B.C. Tobacco. 2nded. New York: Longman, 1981. 764p.

ANUÁRIO BRASILEIRO DO FUMO. Gazeta Santa Cruz, Santa Cruz do Sul. 2009.152p.

ASSOCIAÇÃO DOS PRODUTORES E COMERCIANTES DE SEMENTES E MUDA DO RS APASSUL. Padrões de comercialização de sementes de fumo. Disponível em:

www.apassul.com.br/arquivo/padroesrs/fumo.pdf. Acesso em: 16 jun. 2012.

BARNETT, H.C.; HUNTER, B.B. Illustrated genera of imperfect fungi. 3.ed. Minneapolis: Burgess Publications, 1987. 218p.

DHINGRA, O.D., SINCLAIR, J.B. Basic plant pathology methods. 2. ed. Boca Raton: CRC Press, 1995. 434p.

HUTCHENS, T.W. Agronomy and Physiology: Tobacco Seed. In: Tobacco: production, chemistry and technology. Lexington: Rickard Seeds, p.66-69. (sem data).

LUCCA-FILHO, O.A. Importância da sanidade na produção de sementes de alta qualidade. Revista Brasileira de Sementes, v.7, n.1, p.113-124, 1985.

MEDEIROS, E.M. Maturação Fisiológica e adaptação do teste de envelhecimento acelerado para sementes de fumo. 2008.17f. Tese (Doutorado em Ciência e Tecnologia) Faculdade de agronomia Eliseu Maciel, Universidade Federal de Pelotas.

MILLER, J.D. Fungi and mycotoxins in grain: implications for stored product research. J. Stored Prod. Res., v.31, n.1,p.1-16. 1995

NEERGARD, P. Seed Pathology. London: The Macmillan. 1979. 289p.

SEFRIN, G. O fumo no Brasil e no mundo. Santa cruz do Sul: AFUBRA, 1995.186p. 